\title{
Current breastfeeding practices in Bulgaria
}

\section{Abstract}

The results obtained by the national monitoring of dietary intake and nutritional status of Bulgarian infants, conducted during the last ten years, show some negative characteristics and trends associated with risk of chronic non-communicable diseases and micronutrient deficiencies later in life. The data on nutrition in infants were collected through active interviews with the mothers and information from the medical documentation of their GPs during the surveys in 2007 and 2014. Wrong practices of infant feeding and serious problems in their nutritional status, such as short duration of breastfeeding, low prevalence of exclusive breastfeeding, early and inadequate complementary feeding, high prevalence of stunting, underweight and anaemia among infants, especially in minorities, were identified. Results obtained during the survey in 2007 were: the rate of children breast-fed in the first days after their births were $90.7 \%$. Only $1.8 \%$ was breast-fed during their first hour according to WHO and UNICEF recommendations; $12.5 \%$ of the infants were at exclusive breast-feeding. Data were compared with the results obtained for children at the same age from national surveys conducted in 2014, as the rate of infants who were breast-fed in the first days after their births was $86.3 \% ; 9.9 \%$ were breast-fed during their first hour and $21.7 \%$ of the infants were at exclusive breast-feeding. The total duration of breast-feeding was short according to both surveys. The rate of breast-fed infants in the first days after birth is high in many European countries, however, the breast-feeding practices in Bulgaria do not comply with the international recommendations. The exclusive breast-feeding has a low prevalence rate. The breast-feeding period is very short. It is necessary to develop the national recommendations for healthy nutrition of infants that will concern all these problems and will aim to improve knowledge of the mothers regarding the best practices of healthy nutrition of their infants.

\section{Conflict of Interest}

There is no conflict of interest 